\title{
Journal of Surgery
}

\section{[Jurnalul de Chirurgie]}

\section{Quality of resection in emergency colon cancer surgery - a retrospective study}

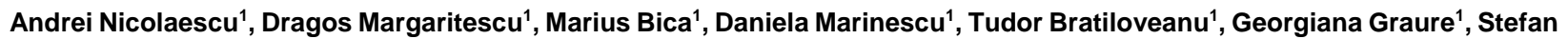 \\ Patrascu', Konstantinos Sapalidis ${ }^{2}$, Valeriu Surlin ${ }^{1}$ \\ ${ }^{1} 6^{\text {th }}$ Surgical Department, University of Medicine and Pharmacy of Craiova, Romania, ${ }^{2} 3^{\text {rd }}$ Surgical Department, Aristotle University \\ of Thessaloniki, Thessaloniki, Greece
}

\begin{abstract}
:
Background. The surgical attitude and the treatment towards colon cancer did not change significantly over the past years. The most important features in achieving oncologically safe surgery are clean resection wedges and the removal of the locoregional lymph nodes. This study aimed to identify if emergency colon cancer surgery has any influence on the type and quality of resections. Methods. We performed a retrospective study of 163 patients admitted in our surgical department with colon cancer who underwent an oncological resection. The patients were divided in two groups: group A included patients that underwent emergency surgery in the emergency and group B included patients undergoing elective surgery. A comparative analysis of intraoperative markers for oncologic quality of resection and for the postoperative outcomes was performed.

Results. Of the 163 patients included in this study, 104 were operated in the emergency setting and 59 as elective cases. The preoperative status of patients indicated that emergency cases had a more precarious nutritional and biological status. When the quality of oncologic resection was analyzed, no differences were observed. A higher number of ostomies were created in group A, as well as worse postoperative outcomes. Conclusion. Emergency surgery for colon cancer did not affect the quality of resection, but the morbidity was higher in patients who presented as surgical emergency.
\end{abstract}

Keywords: colon cancer, surgery, resection

\section{Background}

The surgical attitude towards colon cancer has remained relatively constant. The prime step in obtaining oncologically safe surgery is to have a specimen without tumoral invasion in the resection margins and to remove the locoregional lymph nodes.

In general, colon cancer is relatively straightforward to manage and it provides the surgeon a far better operative field compared to rectal cancer. Despite insufficient evidence, it is conventionally believed that intraparietal bowel invasion rarely exceeds $2 \mathrm{~cm}$, both cranially and caudally, therefore a $5 \mathrm{~cm}$ safety margin is generally enough to allow a good oncologic safety profile. [1,2] In certain instances, obtaining the $5 \mathrm{~cm}$ safety margin might prove difficult without further surgical maneuvers, such as splenic flexure mobilization, further influencing the complexity of the surgical procedure and the postoperative outcome. Other than the tumor-free margins, the vascular supply of the excised colon and the level of vascular ligation are the essential factors involved in the amplitude of the resection. Moreover, it is the adequate excision of the mesocolon and the lymphadenectomy, which are probably the most important aspects for the quality of colon cancer surgery [3].

\footnotetext{
Corresponding author: Stefan Patrascu, Petru Rares Str 2, Craiova 200349, Romania, stef.patrascu@gmail.com

Received April 29, 2021; Accepted May 29, 2021, Published June 29, 2021

Citation: Nicolaescu A., Margaritescu D., Bica M, Marinescu Daniela, Bratiloveanu T, Graure Georgiana, Patrascu Șt, Sapalidis K, Surlin V: Quality of resection in emergency colon cancer surgery - a retrospective study Journal of Surgery [Jurnalul de chirurgie]. 2021; 17 (2): 107-111 DOI: 10.7438/JSURG.2021.02.04
}

Copyright: () 2021. Andrei Nicolaescu1This is an open-access article distributed under the terms of the Creative Commons Attribution License, which permits unrestricted use, distribution, and reproduction in any medium, provided the original author and source are credited. 
The aim of the current study was to assess the impact and the implications of emergency colon cancer surgery on the type and quality of oncologic resections.

\section{Methods}

This cohort study analyzed the data of 163 patients with colon cancer undergoing oncologic resections admitted in a tertiary care center (Emergency County Hospital of Craiova - SCJUC) between January 2018 and December 2020. The study was approved by the SCJUC Institutional Review Board. Patients were classified in two groups: the first group (group A) included patients that underwent emergency surgery in the emergency setting, while the second group (group B) consisted of patients undergoing elective surgery. Data such as age, sex, comorbidities, TNM stage, type of surgery, substitutes for surgical quality, and postoperative outcomes were retrospectively retrieved from the institutional patient data repository. The substitutes for surgical quality of oncologic resections included the tumoral invasion of the proximal and distal resection margins, number of harvested lymph nodes, and number of positive lymph nodes. The postoperative outcomes included the type of postoperative complication, classified according to the Clavien - Dindo scale, anastomotic leaks, type of reintervention, and number of days until hospital discharge. Other variables taken into consideration included tumor stage, size, topography, and histopathologic assessment. The histopathological evaluation comprised of the histopathological subtype, grading, lymph node involvement, tumoral inflammatory infiltrate, microvascular and perineural invasion. Clinical and perioperative variables were categorized using descriptive statistics. T-student test was used for the statistical analysis of quantitative variables. $\mathrm{P}$ of less than 0.05 was considered as statistically significant. The statistical analysis was performed using the SPSS software, version 20.0 (IBM Corp, USA).

\section{Results}

Of the 163 patients included in this study, 104 were operated in the emergency setting and 59 as elective cases. The majority of patients in both groups had rural residence, with patients in the emergency group being younger (69.19 vs 71.68, $\mathrm{p}=0.11)$. The sex ratio $(\mathrm{M} / \mathrm{F})$ was 0.96 in group $\mathrm{A}$ and 1.8 in group $\mathrm{B}$ $(p<0.01)$. The rates of alcohol abuse, smoking, and comorbidity burden did not indicate statistically significant differences.

The preoperative status of patients indicated that emergency cases had a more precarious nutritional and biological status. The mean weight in group A was lower than in group B (76.3 vs 83.6, p=0.23). Although the mean $\mathrm{Hb}$ level in emergency patients was lower than in elective cases, no statistical difference was observed between the two groups (10.25 vs $10.85 \mathrm{~g} / \mathrm{dl}, \mathrm{p}=0.18$ ). However, there was a statistical difference between groups in mean serum protein levels $(6.24$ vs $6.59 \mathrm{~g} / \mathrm{dl}, \mathrm{p}=0.01)$, with the majority of patients in group A (59\%) displaying mild or moderate hypoproteinemia (table 1).

Table 1: Clinico-demographic data of patients undergoing colon cancer surgery in the emergency and elective settings.

\begin{tabular}{r|llc} 
& Group A & Group B & \\
\hline Age & 69.19 & 71.68 & 0.11 \\
Weight $(\mathrm{kg})$ & 76.3 & 83.6 & 0.23 \\
Sex ratio $(\mathrm{M} / \mathrm{F})$ & 0.96 & 1.8 & 0.008 \\
Preoperative Hb $(\mathrm{g} / \mathrm{dl})$ & 10.25 & 10.85 & 0.18 \\
Preoperative serum proteins & 6.24 & 6.59 & 0.01 \\
level $(\mathrm{g} / \mathrm{dl})$ & & $11-22.44 \%$ & 0.29 \\
Metastatic disease (number & $22-21.15 \%$ & &
\end{tabular}

When the quality of oncologic resection was analyzed, no differences were observed in relation to proximal and distal margins of the excised specimen, as all patients had oncologically clear resection lines. Likewise, the differences in mean number of examined and invaded lymph nodes between groups did not reach statistical significance. Significant intraoperative bleeding occurred more frequently in emergency procedures, and the mean blood volume loss was significantly higher in these cases (685 vs. $323 \mathrm{ml}, \mathrm{p}=0.04$ ). Colostomy and ileostomy were more frequently performed in patients from group $\mathrm{A}$ that those in group B (28 vs 4, and 5 vs 0 respectively), with a certain effect on the postoperative quality of life (table 2). 
Table 2: Main intraoperative parameters in emergency and chronic cases.

\begin{tabular}{r|lll} 
& Group A & Group B & $p$ \\
\hline Tumor size $(\mathrm{cm})$ & 5.4 & 4.7 & 0.04 \\
Lymph nodes examined & 13.63 & 11.55 & 0.11 \\
Invaded lymph nodes & 1.81 & 2.04 & 0.71 \\
Positive resection edges & 0 & 0 & - \\
Vascular section at origin & $39(37.5 \%)$ & $44(74.57 \%)$ & 0.03 \\
Intraoperative blood loss $(\mathrm{ml})$ & 685 & 323 & 0.04 \\
/ range) & & & \\
Number of colostomies & 28 & 4 & 0.008 \\
Number of ileostomies & 5 & 0 & -
\end{tabular}

Concerning the surgical intervention in itself, the most commonly used type of surgical procedure for emergency patients was right hemicolectomy, followed by proctosigmoidectomy (Hartmann's operation $-26.92 \%$ ) and left hemicolectomy. In case of elective surgery, right hemicolectomy was also the main option for right-sided colon cancers $(42.37 \%)$, followed by rectosigmoidectomy $(23.72 \%)$. No laparoscopic approach was attempted in the emergency setting, whereas $35 \%$ of patients were operated by minimally invasive surgery in elective cases (table 3 ).

Table 3: The specific type of procedure performed in emergency and elective cases

\begin{tabular}{l|ll} 
& Group A & Group B \\
\hline Right hemicolectomy & $47(45.19 \%)$ & $25(42.37 \%)$ \\
\hline Left hemicolectomy & $19(8.26)$ & $6(10.16 \%)$ \\
\hline Sigmoidectomy & $14(13.46 \%)$ & $11(18.64 \%)$ \\
\hline Rectosigmoidectomy & $12(11.53 \%$ & $14(23.72 \%)$ \\
\hline Hartman resections & $28(26.92)$ & $4(6.77 \%)$ \\
\hline Segmentary resection & $5(4.8 \%)$ & $3(5.08 \%)$ \\
\hline $\begin{array}{l}\text { Extended resections (stomach, small } \\
\text { bowel) }\end{array}$ & $3-2.88 \%$ & 0 \\
\hline Laparoscopic approach & 0 & $21-35 \%$ \\
\hline
\end{tabular}

Table 4: Postoperative outcomes in colon cancer patients undergoing emergency vs elective surgery

\begin{tabular}{r|lll} 
& Group A & Group B & $p$ \\
\hline Postoperative diuresis (ml) & 1588 & 1827 & 0.056 \\
first 24h) & & & \\
Postoperative ileus (days) & 5.5 & 3.1 & 0.04 \\
Anastomotic fistula & 7 & 4 & 0.02 \\
Postoperative complication & & & \\
(Clavien Dindo) & & $36(61 \%)$ & 0.22 \\
No complication & $57(54.80 \%)$ & $3(5.08 \%)$ & 0.3 \\
1 & $4(3.84 \%)$ & $12(20.33 \%)$ & 0.08 \\
2 & $12(11.53 \%)$ & $5(8.47 \%)$ & 0.06 \\
3 & $10(9.61 \%)$ & $1(1.7 \%)$ & 0.02 \\
4 & $6(7.69 \%)$ & $2(3.38 \%)$ & 0.01 \\
5 & $15(14.42 \%)$ & 15.98 & 0.16
\end{tabular}

Several postoperative outcomes, such as the postoperative ileus, Clavien-Dindo grade 4 and 5 complications, and the anastomotic leak rate, displayed significantly higher values in group A than in group B. The mean volume for the $24 \mathrm{~h}$ postoperative diuresis was lower in emergency cases, displaying a trend toward statistical significance (1588 vs $1827 \mathrm{ml}, \mathrm{p}=0.056$ ) (table 4). The mean hospital stay 
was longer in case of emergency surgery than in elective cases, without reaching statistical significance (17.73 vs. $15.98, \mathrm{p}=0.16$ ).

\section{Discussion}

For most colorectal cancers, radical surgery with curative intent is the therapeutic gold standard. The main principles are the surgical excision of the tumor, its major vascular supply and the corresponding lymphatic drainage, together with any nearby structure attached to the tumor. The results is a tumor-free margin of resection, which should prevent, at least in theory, any perianastomotic recurrence. It is advisable that at least a $5 \mathrm{~cm}$ safety margin should be obtained in order to avoid such undesired oncologic outcomes. However, it is the locoregional recurrences that are more frequently encountered, usually a consequence of suboptimal lymphadenectomy [5-7]. This is why any clinically suspicious lymph nodes outside the field of resection should be excised and sent for histopathologic examination, in order to determine the correct stage and the proper adjuvant regimen $[8,9]$. At least 12 lymph nodes should be examined to accurately define nodal involvement [10].

Moreover, as the extent of resection in colon cancer surgery is based primarily on the vascular and lymphatic distribution, and knowing the fact that the margins of resection in this case usually exceed the oncological safety margins, it is generally common to have a $10 \mathrm{~cm}$ resection margins due to vascular concerns. However, a better, yet more complex approach would take into account aspects such as tumoral staging, grading, or histologic subtype [11$13]$.

On the other hand, the assessment of the quality of resection in emergency colon cancer surgery is important because of the advanced stages in which these tumors are diagnosed, their precarious nutritional and immunologic status, which lead to poor postoperative outcomes and reduced survival rates [14]. Furthermore, the extent of resection in colon cancer surgery also varies with the topography of the primary tumor. Sometimes, in case of patients with colon polyposis or familial colon cancer, the surgeon may attempt total colectomy or proctocolectomy in order to reduce the risk of metachronous cancer [1517]. Too often, emergency colon cancer surgery may offer unwanted surprises to the operator, such as invasion in the surrounding organs or liver metastasis, making a proper resection even more cumbersome. Moreover, the extent of the resection should be adjusted to age and associated pathologies, which may limit the therapeutic options, especially in case of emergency cases.
Our study indicates that the emergency setting in itself does not significantly alters the quality of resection, but will lead to worse immediate postoperative outcomes, including higher postoperative ileus and complication rates, increased incidence of ostomy creation, and longer hospital stay. One major drawback of this study, other than its retrospective nature, is the lack of long-term postoperative follow-up, which could have offered more data on the quality of life and recurrence rates.

\section{Conclusions}

The quality of resection was not influenced by the emergency indication for surgery in respect to the main parameters considered, while the postoperative morbidity was higher in patients operated in the emergency setting. However, given the retrospective nature of this study, additional research is required to draw definitive conclusions concerning the perioperative oncologic outcomes in emergency colon cancer surgery.

\section{Institutional Review Board Statement:}

The study was conducted according to the guidelines of the Declaration of Helsinki and approved by the Institutional Ethics Committee of the Emergency County Hospital of Craiova (no. 68 / 28.04.2021).

\section{Informed Consent Statement:}

Informed consent was obtained from all subjects involved in the study.

\section{Disclosure:}

The authors have no financial ties to disclose.

\section{References}

1. Grinnell RS. Distal intramural spread of carcinoma of the rectum and rectosigmoid. Surg Gynecol Obstet. 1954; 99:421-30.

2. Quirke P, Durdey P, Dixon MF, Williams NS. Local recurrence of rectal adenocarcinoma due to inadequate surgical resection: histopathological study of lateral tumour spread and surgical excision. Lancet. 1986; 2:996-9.

3. West NP, Hohenberger W, Weber K, Perrakis A, Finan PJ, Quirke P. Complete mesocolic excision with central vascular ligation produces an oncologically superior specimen compared with standard surgery for carcinoma of the colon. J Clin Oncol. 2010; 28:272-8. 
4. Shimada Y, Takii Y, Maruyama S, Ohta T. Intramural and mesorectal distal spread detected by whole-mount sections in the determination of optimal distal resection margin in patientsundergoing surgery for rectosigmoid or rectal cancer without preoperative therapy. Dis Colon Rectum. 2011; 54:1510e1520.

5. Nelson H, Petrelli N, Carlin A. et al. Guidelines 2000 for colon and rectal cancer surgery. J Natl Cancer Inst. 2001; 93:583-96.

6. Poon RT, Fan ST, Ng IO, Wong J. Significance of resection margin in hepatectomy for hepatocellular carcinoma: a critica lreappraisal.Ann Surg. 2000;231:544e551.

7. Chang GJ, Rodriguez-Bigas MA, Skibber JM, Moyer VA. Lymph node evaluation and survival after curative resection of colon cancer: systematic review. J Natl Cancer Inst. 2007;99(6):433-441.

8. National Comprehensive CancerCenter Network Clinical Pratice Guideline in Oncology (NCCN Guidelines): Colon Cancer. Version 3. 2013. [8 August 2013]

9. Edge SB, Compton CC. The American Joint Committee on Cancer: the 7th edition of the AJCC cancer staging manual and the future of TNM. Ann Surg Oncol. 2010;17(6):14711474.

10. Lindmenn F, Schlimok G, Dirschedl P. et al. Prognostic significance of micrometastatic tumor cells in bone marrow of colorectal cancer patients. Lancet. 1992; 340:685-9.

11. Siegel R, Ward E, Brawley O, et al. Cancer statistics, 2011: the impact of eliminating socioeconomic and racial disparities on premature cancer deaths. CA Cancer J Clin $2011 ; 61: 212-36$
12. Schmoll H.J., Van Cutsem E., Stein A., Valentini V., Glimelius B., Haustermans K., Nordlinger B., van de Velde C.J., Balmana J., Regula J., et al. ESMO Consensus Guidelines for management of patients with colon and rectal cancer. A personalized approach to clinical decision making. Ann. Oncol. 2012;23: 2479-2516. doi: 10.1093/annonc/mds236.

13. Barresi V, Reggiani Bonetti L, Ieni A, Domati F, Tuccari G. Prognostic significance of grading based on the counting of poorly differentiated clusters in colorectal mucinous adenocarcinoma. Hum Pathol. 2015;46: 1722-9.

14. Angelis A, Montibeller G, Hochhauser D, Kanavos P (2017) Multiple criteria decision analysis in the context of health technology assessment: a simulation exercise on metastatic colorectal cancer with multiple stakeholders in the English setting. BMC Med Inform Decis Mak 17(1): 149.

15. Huang JL, Zheng ZH, Wei HB, Huang Y, Chen TF, Wei B. Laparoscopic total colectomy and proctocolectomy for the treatment of familial adenomatous polyposis. Int J Clin Exp Med. 2015;8(6):9173-9176.

16. Watanabe Y, Sato M, Kikkawa H, Yoshida M, Kusunose H, Kawachi K. Hand-assisted laparoscopic total colorectal resection for familial adenomatous polyposis with coexisting rectal cancer. Surg Endosc. 2001; $15: 445-447$

17. Giardiello FM, Allen JI, Axilbund J E, Boland CR, Burke CA, Burt RW, et al. Guidelines on genetic evaluation and management of Lynch syndrome: a consensus statement by the US Multi-Society Task Force on colorectal cancer. Gastroenterology. 2014; 147:502-26. 